\title{
Assessing the Effectiveness of Direct Gesture Interaction for a Safety Critical Maritime Application
}

\author{
Frøy BIRTE BJøRNESETH ${ }^{\mathrm{A}}$, MARK D. DUNLOP ${ }^{\mathrm{B}}$ AND EVA HORNECKER ${ }^{\mathrm{B}}$
}

${ }^{A}$ Rolls-Royce Marine AS, Common Control Platform, Parkgata 3, 6003 Ålesund, Norway. Email: froy.bjorneseth@rolls-royce.com

${ }^{B}$ Computer and Information Sciences, University of Strathclyde, Glasgow G1 1XH, Scotland, UK.Email:mark.dunlop@cis.strath.ac.uk,eva.hornecker@cis.strath.ac.uk

\begin{abstract}
Multi-touch interaction, in particular multi-touch gesture interaction, is widely believed to give a more natural interaction style. We investigated the utility of multi-touch interaction in the safety critical domain of maritime dynamic positioning (DP) vessels. We conducted initial paper prototyping with domain experts to gain an insight into natural gestures; we then conducted observational studies aboard a DP vessel during operational duties and two rounds of formal evaluation of prototypes - the second on a motion platform ship simulator. Despite following a careful user-centred design process, the final results show that traditional touch-screen button and menu interaction was quicker and less erroneous than gestures. Furthermore, the moving environment accentuated this difference and we observed initial use problems and handedness asymmetries on some multi-touch gestures. On the positive side, our results showed that users were able to suspend gestural interaction more naturally, thus improving situational awareness.
\end{abstract}

\section{Introduction}

The maritime field is deeply rooted in tradition but has recently experienced an explosion of technological development from maritime equipment suppliers. The rapid advancements in technology, particularly automation systems, has led to increasingly complex and varied user interfaces. An operator must interact with many different systems, often with different interface styles, during an operation. On Dynamic Positioning (DP) vessels, which is the key focus of our work, the operator role can become stressful as (s)he must interact with at least three different systems - each with its own graphical user interface (GUI) and display. In addition, (s)he must lead the radio communication, have an eye on the propulsion system and maintain constant observational awareness of the environment around the vessel. This can be a challenge both mentally and physically, and operators' cognitive load can increase if presented with too much information (Lazet and Schuffel, 1977). Physical strain also affects the operator if the equipment is poorly ergonomically placed (Galliers et al., 1999). 
Many of the displays on a modern vessel's bridge utilised to control the vessel are currently operated via single-touch interaction that require precise finger accuracy to select buttons in a graphical interface, which can be a challenge in rough sea conditions. In this paper we report our detailed investigation into using multi-touch gesture interaction to control systems onboard offshore vessels with the aim of reducing interaction focus and thus increasing situational awareness for the operator, particularly during potentially dangerous operations.

Multi-touch is hypothesised to improve interaction (see e.g. Buxton (2007): bi-manual or multi-finger interaction is more efficient than one control point). We compared multi-touch gesture interaction, using a gesture set developed through a guessability study (Wobbrock et al., 2009), with traditional button and menu-based touch interaction to control the commercially available Rolls-Royce Marine DP system in a moving versus a static environment. So far, there has been little research on the use of multi-touch gesture interaction within safety-critical domains. On-board a marine vessel as with other safetycritical industries such as nuclear, transport and aviation, productivity, efficiency and user satisfaction are important factors but safety always remains the overriding concern (MacKay, 1999). DP systems are complex and often controlled using single touch displays in combination with physical input devices such as joysticks. Users interact with these singletouch displays via discrete operations, touching buttons and tapping or double-tapping. Our research investigates whether direct manipulation of the GUI using multi-touch gesture interaction (continuous operations) can increase the DP operator's situational awareness, enhance the feeling of control, and improve ease of use and speed of interaction by omitting complex menu navigation.

Our hypotheses are that, when compared with traditional menu/button interaction, in DP systems:

- H1: Multi-touch gesture interaction will lead to faster interaction.

- H2: Multi-touch gesture interaction will be less erroneous.

- H3: Multi-touch gesture interaction will lead to more situational awareness.

- H4: Multi-touch gesture interaction will be less affected by a moving environment.

Our first study investigated potential gestures that users find intuitive for controlling the Rolls-Royce DP system. Given this is a specialist interface, we had expert users (DP operators and designers) suggest gestures they would find intuitive through paper prototyping (Bjørneseth et al., 2008). We identified the most common gestures that could be implemented consistently without ambiguities. To gain a better understanding of the domain and the cognitive and manual demands during DP operation work we conducted an observational 
study on-board an offshore vessel in the North Sea during real operations, focusing on system/interface interaction.

In addition to the initial gesture-elicitation study, two working prototypes that implemented the identified gestures were developed for multi-touch displays, with two iterations of testing to refine the gesture set (using a mix of DP experts and maritime students). Testing was conducted using an industrial DP system from Rolls-Royce Marine (with simulated control data) to compare the efficiency of continuous multi-touch gestures against the standard button-and-menu interface (controlled via discrete touch-screen actions). To explore the viability of touch gestures in a maritime environment, we investigated the impact of ship motion on interaction by running the final study on a motion platform that simulates a ship in rough sea.

\section{Background and Related Research}

We first describe the background of our research in the maritime domain and how the human element affects the work environment on-board a vessel in terms of safety-critical systems. Then we summarize research on multi-touch gesture interaction, focusing on work directly relevant for our project.

\subsection{The Marine Environment: DP Operation as a Safety-Critical Component}

Our work focuses on the use of DP systems, which enable a vessel to maintain position without anchoring. DP systems are crucial to all offshore operations within the oil and gas industry. In addition, they are used on yachts and cruise ships whenever these have to dock at places where it is not possible to anchor (e.g. over vulnerable sea-beds such as coral reefs, or very deep sea). We describe the safety-critical nature of the work situation, and give an overview of how the human element is taken into account during design of equipment and systems.

\subsubsection{Dynamic Positioning Systems}

A DP system (Bray, 2003) is defined as: "A computer controlled system to automatically maintain a ship's position and heading by using her own propellers and thrusters." The system is based on algorithms that automatically compensate for wind, waves and currents. 


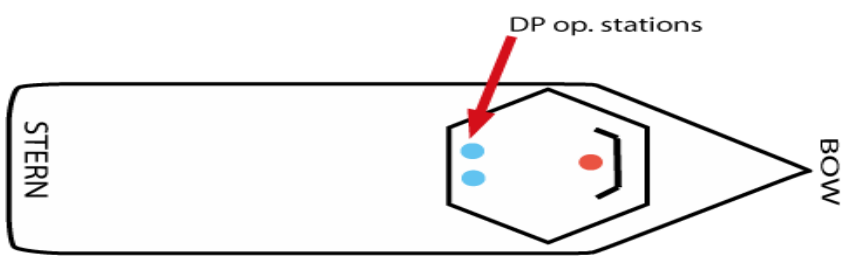

Figure 1: Overview of vessel with DP op.stations on the aft bridge

The bridge of an offshore vessel is divided into fore and aft bridge. The fore bridge (bow facing) has the traditional captain's chair (facing forward and utilised when steaming), while the aft deck (stern facing) holds the DP operator station (see figure 1 and 2) and other equipment used during DP operation (e.g. winch operation equipment). The windows on the aft bridge go from floor to roof and are the operator's main view to supervise operations carried out on the aft deck by the deck hands. Therefore it is crucial that nothing occludes the operator's view out of the windows. Additionally, even small deviations in the vessel's position during safety-critical operations can cause serious accidents and damage to both vessel and offshore installations.

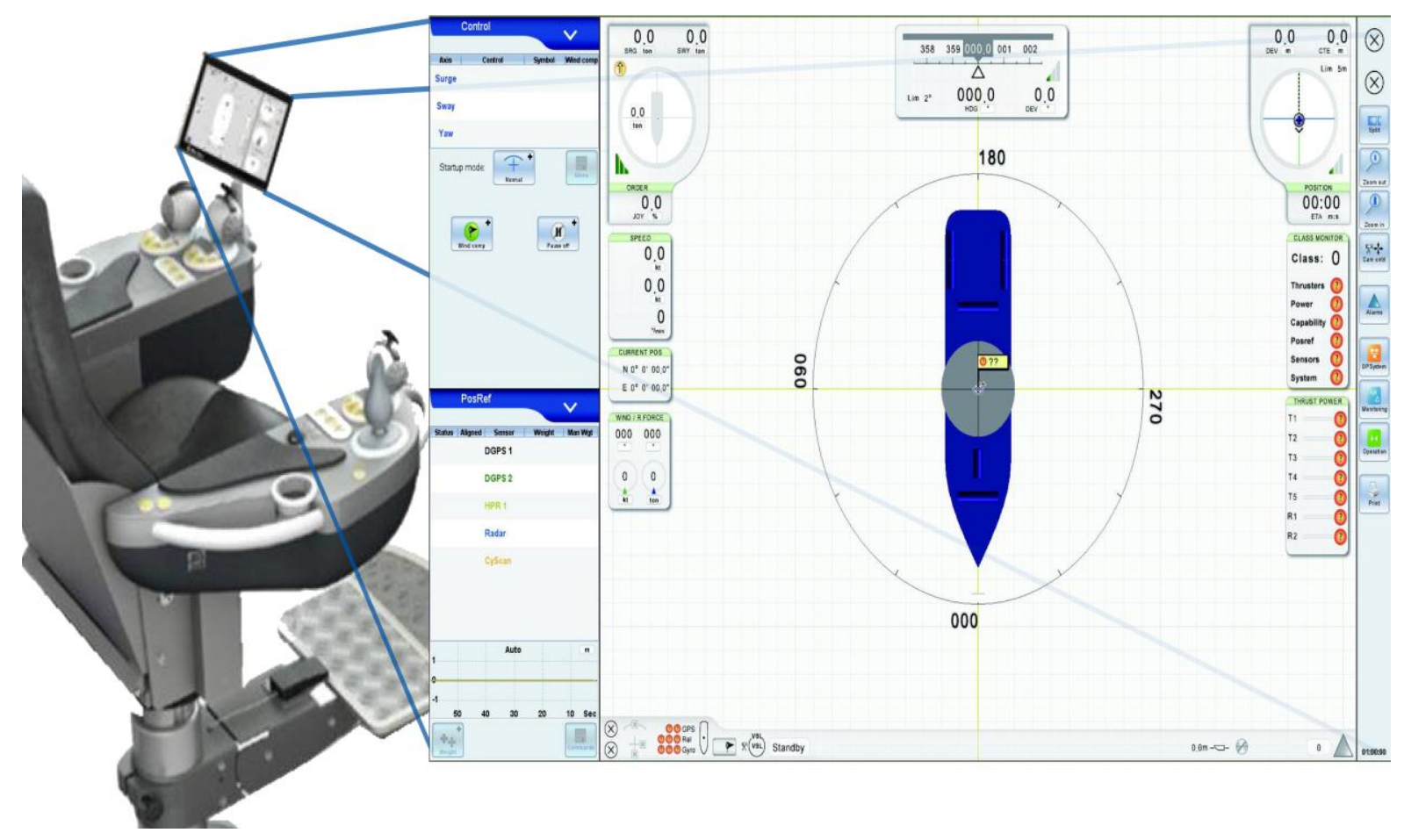

Figure 2: Rolls-Royce DP operator station and traditional interface with menus and buttons

Figure 2 shows the Rolls-Royce DP system. This shows a 2.5D image of the vessel, and is operated on a single-touch screen by selecting from menus and buttons. Operation peak time is when the vessel is closing in on an oilrig to position itself next to it to load or offload liquids and cargo, and when the vessel withdraws after the scheduled tasks have been completed. This will be further explained in section 4. During this period of time the operator sits in the operator chair and enters values into the system using input devices such as a joystick and propulsion controls. To enter values the operator has to tap the blue arrow in the left hand menu and select the correct menu item (Change). A panel appears showing different 
sub-selections that concern the type of position change wanted. The operator selects the most suited by double tapping the menu item and a soft number keypad appears. The operator now enters the values, waits for the system to respond (approximately 1 second) and then presses Apply. The vessel will then automatically start to move in the set direction.

The Rolls-Royce DP system manipulates surge, sway and yaw movements of the vessel's six available degrees of freedom (figure 3), with the marine environment affecting heave, roll and pitch (though zooming the scene in the interface is often also referred to as heave). The system must be closely monitored and the graphical user interface (GUI) interpreted by the operator with tasks carried out efficiently (Mills, 2005). The feeling of control during operations is maintained through a combination of sensing the ship (Prison, Lützhöft, Porathe, 2009) and monitoring the sometimes complex information presented in the visual display units (VDU).

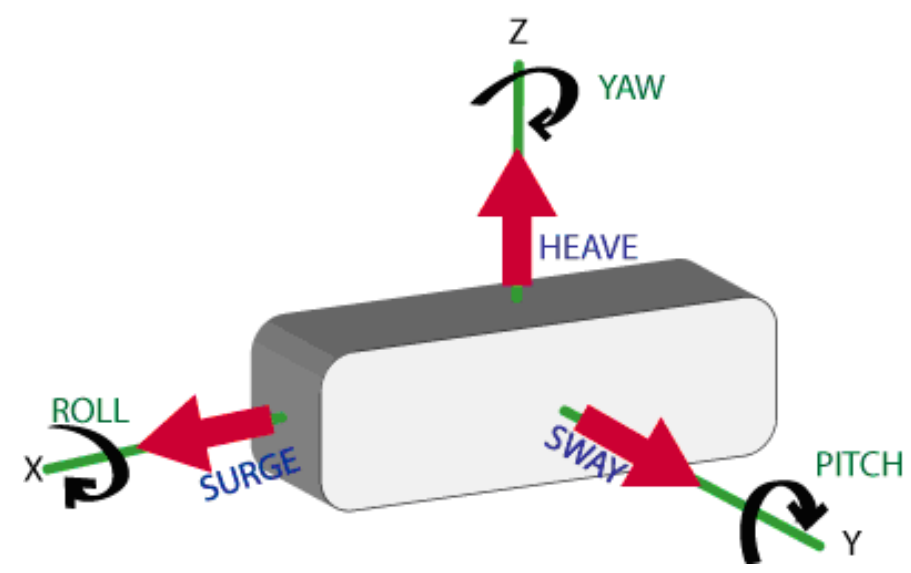

Figure 3: Degrees of freedom

\subsubsection{The Human Element On-board}

Generally poor usability causes fatigue and strain in operators and can lead to loss of attention or accidents (Nielsen and Jungnickel, 2003). According to Lloyd's Register's World Casualty Statistics (Fairplay, 2007), there has been a decrease in total vessel losses in the period 1995 to 2007, however there has been a concerning increase in lives lost as a result of total loss incidents. As a result the human element has recently received more attention in the design and development of new equipment and vessels.

The 'human element' refers to the interaction between a human and any other human, system or machine aboard a ship (Squire, 2009). Unfortunately, human factors are rarely considered in vessel design and engineering even though it is estimated that $80 \%$ of accidents have a human cause (Shea and Grady, 1998). The main industry focus is cost-efficiency, with the crew rarely involved in the design process. This can cause poorly adapted equipment and increased cognitive load during standard operation. If stress levels increase during safetycritical situations, cognitive load rises further, and the operator may fail to take the right 
decisions. According to Redmill and Rajan (1997) safety is a state in which human life and well-being and the environment are not endangered.

What differentiates industrial technology from consumer technologies is often these safetycritical issues that can significantly slow down the adoption of new technology in what Sillitoe, Walker and Earthy (2009) refer to as a conservative industry. While shipyards and ship owners do try to maintain safety as a priority, often the safe and well-known only provides a false security and avoids spending on innovative research.

Reason (1990) discerns active failure and latent failure. Latent failure, frequently seen onboard vessels today, originates from preceding actions, involves working conditions and load, competing demands, and is caused by designers, developers, decision-makers and managers. There are two main approaches to handle the problem of human error (Song, 2009): increasing the number of well-trained crew or improve the working environment on-board. The latter is a more long-term solution that solves the actual problem and leads us into the field of usability and human machine interaction, where our research aims to contribute. The operator's only possibility of interaction and manipulation of the DP system is through its interface consisting both the physical equipment and the visual presentation and interaction, the GUI.

\subsection{The Potential for Multi-touch}

While multi-touch gesture interaction has been researched since as far back as 1982 (Mehta,1982) as discussed by Buxton (2007), it became highly popular through Jeff Han's work and his TED keynote (e.g. Han, 2005). This natural and fluid interaction style inspired us to investigate whether direct gesture interaction might increase efficiency, safety and the feeling of control during maritime operations.

Kirk et al (2009) argue that Fitzmaurice et al's (1997) arguments about spatial multiplexing etc. hold for touch screens just as well as for graspable interfaces. Fitzmaurice showed that having two control points that can be controlled simultaneously to turn a graphical object is much faster than sequential operation. This is supported by research that emphasizes the efficiency of multi-touch and direct gesture interaction. Hancock et al. (2007) presents a project where an algorithm provided 2 DOF's for each touch-point. With three touches, six DOFs could be implemented, and a higher number of touches resulted in an improvement both for performance and user preference. Also, pioneering research by Lee et al. (1985) and Buxton and Myers (1986) shows that the technology provides benefits in terms of interaction. Within maritime research, Mills (2005) emphasises that in safety critical situations, button press combinations can be very hard to remember. In the maritime domain it is important to consider the cognitive load on the operator. Nishino et al. (1997) designed an interactive twohanded gesture interface, and tested a range of gestures. They found increased efficiency for using two hands, but in some cases the rate of recognition was too low and the test subjects 
were confused by the variety of gestures available. This means that the number of gestures has to be limited.

The current DP interface requires a rather lengthy series of menu-button interactions, which is difficult to learn. Direct gesture manipulation promises a more straightforward means of input. It can exploit direct mappings between desired ship movement and manual input (move left, right, up, down, rotate/turn at an angle etc.). This could benefit DP operation by being more intuitive and less error-prone (e.g. a lower risk of confusing menu items or the directionality of movement, currently done by pressing a button for the non-default direction), reducing cognitive load, and being quicker than the current system. Furthermore, it is important to note that during DP operations, the main action happens on the aft deck of the vessel. The operators spend the majority of their time looking out the windows for safety reasons to make sure that the vessel is stable for the aft deck activities (loading/offloading etc.). This will be further explained in section 4. It would therefore be beneficial if the operators could operate the system by only glancing down, with almost eyes-free interaction, instead of carrying out complex menu navigation. On the other hand, direct touch input is known to have disadvantages, such as the 'fat finger problem', accuracy (difficulty of selecting small targets and doing precise positioning), and occlusion of the target object by the hand (Forlines, 2007). These issues might cancel out the advantages hoped for. In terms of commercial value, Rolls-Royce Marine aim to be leading in terms of state-of-the-art technology usage and were interested in investigating possible improvements of the already successful Rolls-Royce DP system to enhance the user experience.

Our and Rolls-Royce Marine's interest thus was to explore the potential for multi-touch gesture interaction to improve DP operation using the commercially available Rolls-Royce DP system. Given some of the DP tasks correspond with image manipulations typical for multi-touch (zoom and rotate), we decided to focus on multi-touch gesture interaction. What distinguishes our research from that on consumer electronics such as Microsoft's Surface and the iPhone is that, if successful, these interaction mechanisms would be introduced into a new generation of technology used in safety-critical maritime environments.

\section{Devising a User-Generated Gesture Set for DP Control}

Our first study consisted of a guessability experiment with eight participants and resulted in a user-generated set of gestures. The aim of this study was to investigate which gestures felt natural to participants for operating a touch screen DP system. Guessability studies (Nielsen et al., 2003, Wobbrock et al., 2009) are a user-centred design method for generating gesture sets that are easy to perform and remember, intuitive, and adequate to the task. Users are asked to suggest gestures for a given set of elemental core operations or tasks. From these, the researcher extracts the most common gestures while eliminating overlaps or gestures that might be difficult to perform or not detectable by the system. User-defined gestures tend to be simpler then the gestures invented by designers (Morris et al., 2010) however, it is an open 
research question whether generic (non-application-specific) gestures or application-specific gestures are superior (cf. Micire et al., 2009).

\subsection{Study Design}

Given the specialist and safety-critical nature of the task, we involved domain experts and used a domain-specific representation instead of relying on generic gestures. This study is described in detail in Bjørneseth, Dunlop and Strand (2008).

As visual trigger for participants and for them to show suggested gestures we used a paper prototype. This emulated the graphical user interface of the DP system and allowed participants to move a blue cardboard vessel on the paper surface. Normally the main DP operator-display is placed vertically to the left side of the operator (see figure 2). In this case, the prototype display was placed in a desk-like position in front of the operator (see figure 3), adjusted to suit usage of both hands. The study was conducted using a $2 \mathrm{D}$ representation of the vessel, in contrast to the 3D environment available in the real-life system.

Our expert participants did not hold DP certificates, but had extended knowledge of DP from developing DP systems and manoeuvring vessels during sea acceptance trials, where the DP system undergoes fine-tuning to the vessel's characteristics. Table 1 summarises participants' background - no noticeable differences in performance were identified between these categories.

\begin{tabular}{|r|l|l|}
\hline Age & 6 users 24-44 & 2 users 50+ \\
\hline Gender & 7 male & 1 female \\
\hline Title/education & $\begin{array}{l}\text { 6 DP software developers } \\
\text { with MSc, BSc }\end{array}$ & $\begin{array}{l}2 \text { Technical Product } \\
\text { Managers (MSc, 50 +) }\end{array}$ \\
\hline
\end{tabular}

Table 1: Overview of participants

The participants were encouraged to move the vessel in any way they found natural, using one or two hands or touching the prototype display at more than one point. They could read through the tasks in advance, but did not have the opportunity to practice.

We investigated manipulation of the three main degrees of freedom (yaw, surge and sway) plus main camera actions. The meaning behind main camera actions in this case refers to the virtual camera in the software, hence you can move the view angle (pan and zoom). Participants were given nine tasks in randomized order, to avoid learning effects, with the vessel returned to its initial position after each task. The tasks concerned moving the vessel a ship's length forward/backward (surge), left/right (sway) and to rotate 
clockwise/anticlockwise by $90^{\circ}$ and $180^{\circ}$ (yaw). In addition, the participants were asked to suggest gestures for actions "heave", "roll" and "pitch".

Each session lasted approximately 10 to 15 minutes including briefing and a post-task discussion. A video camera was used to record hand movements and gestures.

\subsection{Findings}

We observed extensive use of the index finger of the right hand. All participants were righthanded and the majority used their right hand index finger (RI) and the right thumb for most tasks. With little variation the same fingers were used for surge and sway movements: participants pushed the vessel by placing either their dominant index finger alone on the vessel or accompanied by the thumb as a support (see figure 4).
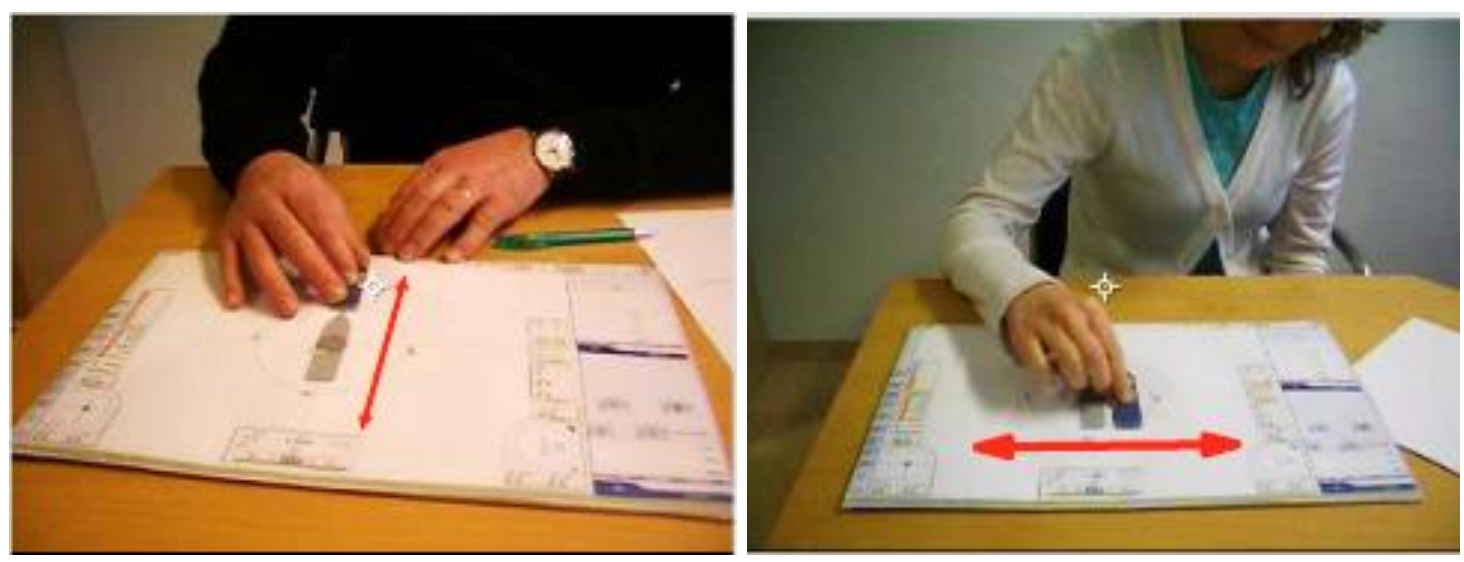

Figure 4: Typical surge and sway interactions

For rotating the vessel there was more variation of techniques: four participants changed method between tasks due to problems in rotating $180^{\circ}$ where the hand gets into an awkward position. The participants could rotate by $90^{\circ}$ using only one hand, whereas the $180^{\circ}$ rotation task was either done in two separate steps using one hand $\left(90^{\circ}+90^{\circ}\right)$ or by using two hands and both index fingers to rotate $180^{\circ}$ in one movement (see Figure 5). 


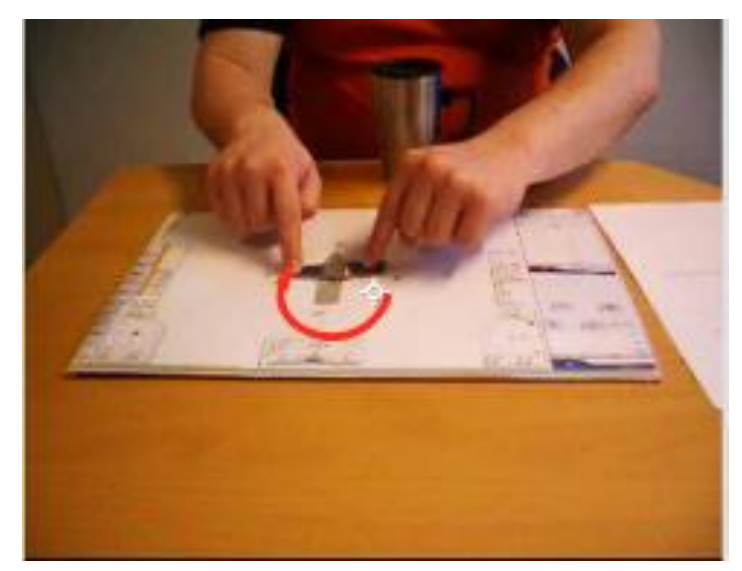

Figure 5: Two-handed yaw interaction (rotation)

To zoom the scene (aka heave) the pinch gesture (known from iPhone) was popular and the observed gestures imply a close relation between pinching the fingers and diagonal slide across the surface. The majority (5 participants) preferred the pinch or a corresponding diagonal slide (figure 6), while the remaining three suggested different movements.
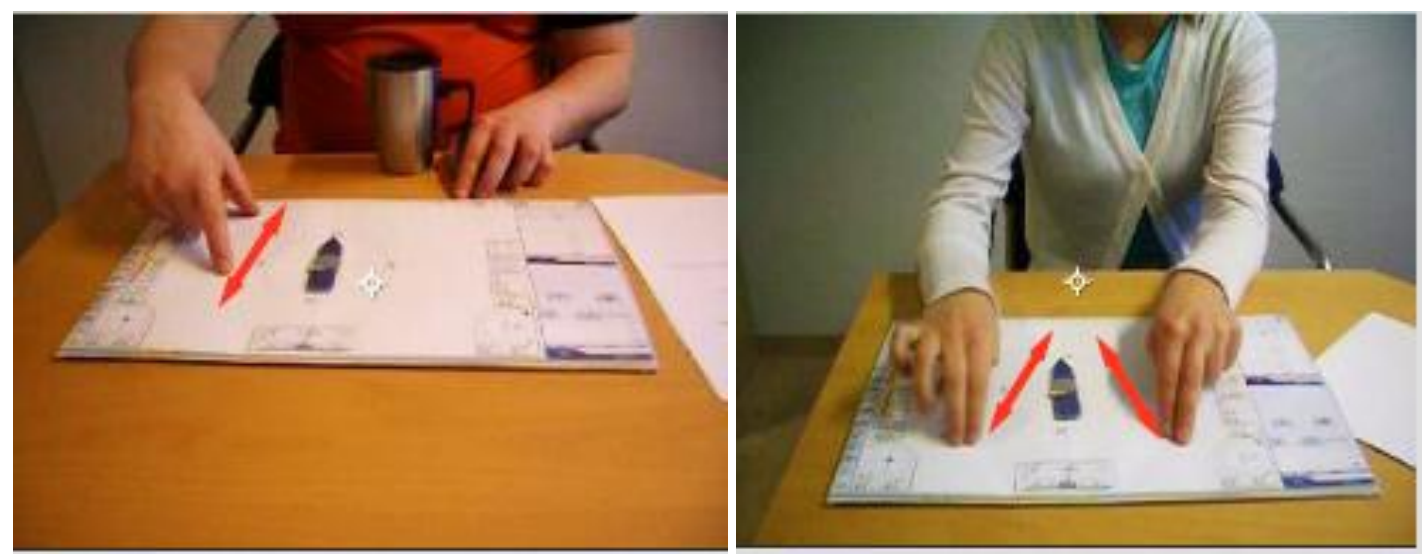

Figure 6: Alternative gestures for zooming (heave)

To rotate the scene around the y-axis (c.f. pitch), half of the participants suggested a vertical curved gesture using their right index finger (figure 7). Others suggested various gestures, some overlapping with gestures suggested for zooming (discarded as ambiguous). Overall, the vertical curve appeared the most natural choice for most users.

Gestures suggested for rotation around $\mathrm{x}$ (roll) were similar to pitch but using a horizontal curve. Three participants indicated that the horizontal curve around the $\mathrm{x}$-axis was the best alternative and two suggested an ambiguous vertical curve around the y-axis. The two remaining participants suggested other gestures. 


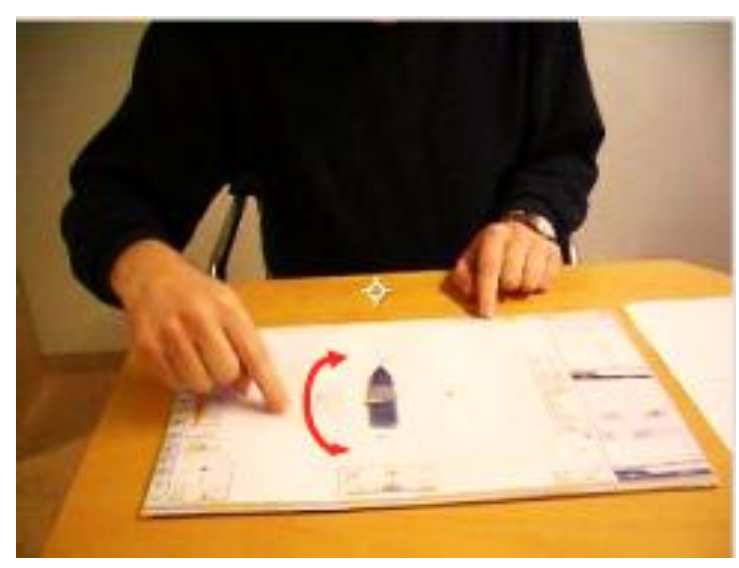

Figure 7: Example pitch gesture

\subsubsection{Post-task Discussion}

The post-task discussion gave insight in participants' concerns on using gestures to operate the DP system. Overall, participants' opinions were positive, especially when using dual or multiple input points. A concern arose around the paper prototype display being placed in front of the operator instead of to the left or right where it is placed in the real system and thus reducing the operator's attention on the aft area of the vessel.

\subsection{Study Discussion and Conclusions}

A set of common, non-ambiguous gestures were identified (table 2). The dominant index finger was used for simple movements with rotations and zooms being performed with two fingers. It is worth noting that for simple movement operations users consistently used single finger gestures while for zooming scene (heave) and rotating the vessel (yaw) they used the clearly distinguishable double finger gestures. To differentiate pitch and yaw these were done away from the vessel on the display. The experiences gained from this first study and participant discussions informed a study carried out on board a platform supply vessel in the North Sea with a refined gesture set implemented in prototype systems discussed later. 


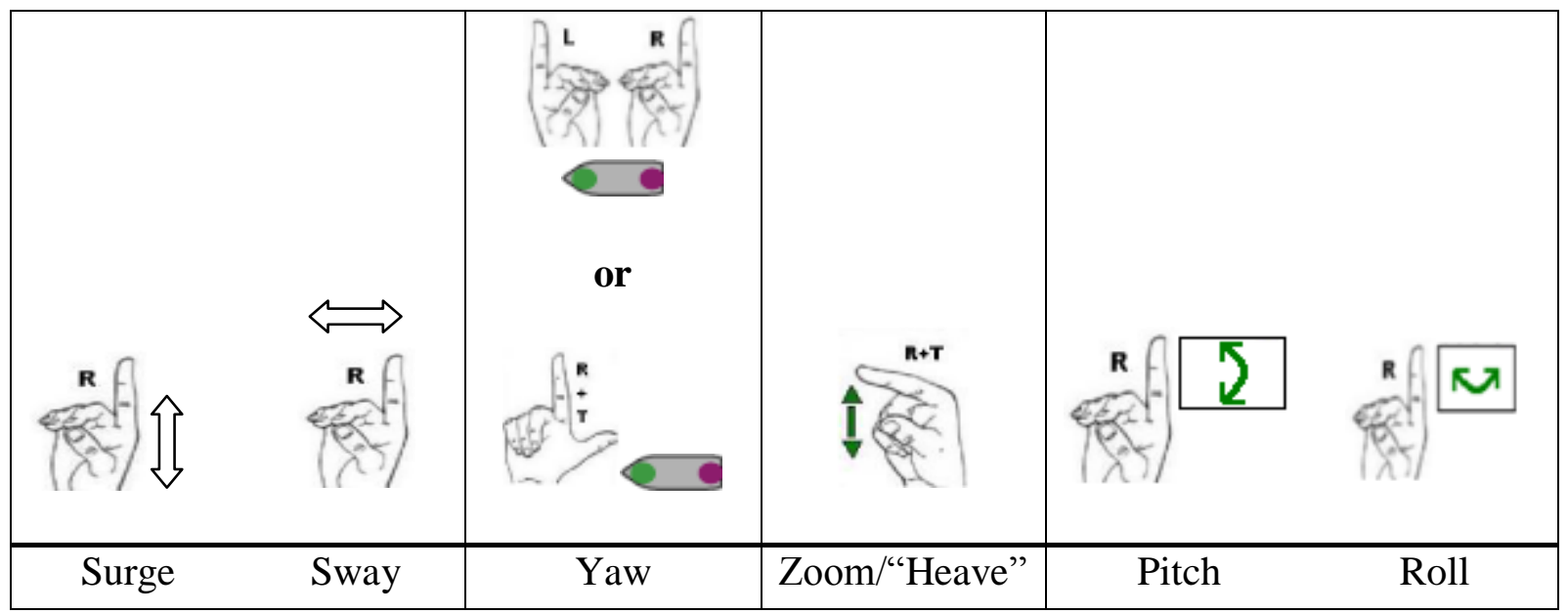

Table 2: Resulting set of gestures

\section{Observations of on-vessel live DP operations}

We conducted an observational study to investigate how DP-operators operate the system in real working environments. The study was conducted over three days in early February on the Platform Supply Vessel (PSV) Havila Foresight (figure 8). The study was informed by the guidelines from Jordan and Henderson (1995) on interaction analysis and Bryman's (2008) book 'Social Research Methods'.

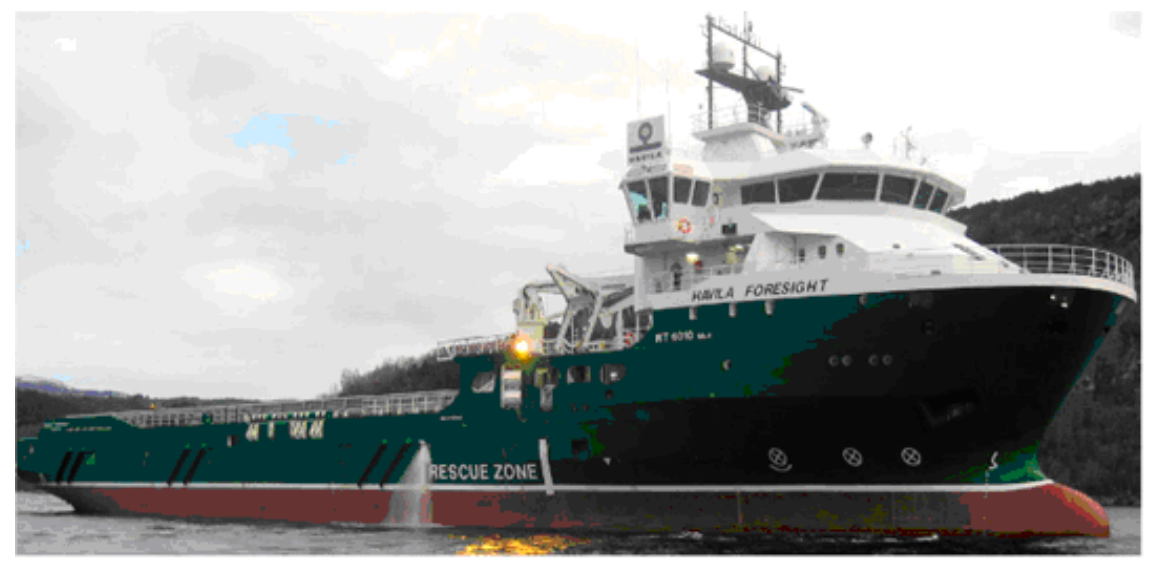

Figure 8: PSV Havila Foresight

\subsection{Study Design}

The crew of the vessel included DP-operator(s), captain, officers, midshipmen, engineers, cook and deck crew - in total 5 qualified DP operators. The vessel's tasks for the three day period were to deliver drilling equipment, food and liquids to four platforms situated in the Norwegian sector of the North Sea offshore between the supply base at Mongstad $(66 \mathrm{~km}$ north of Bergen) and Stavanger (207 km south of Bergen). The crew was observed during some DP operations and when steaming to the oilfield, between platforms and from the oilfield going back to shore. We identified early that the planned DP operations were very similar and conducted throughout the day and night by crew working in shifts. We agreed to 
observe four out of seven scheduled DP operations (one video recorded), including one night operation.

We used two video cameras for recording, one aimed at the operator's face and another behind them recording the operator's movements and the equipment used (joystick, touch displays, emergency switches etc.). Concurrent notes were written with key events noted (e.g. visual sign of operator's mental state) and time stamped. Figure 9 shows the bridge layout and video camera locations. We also handed out questionnaires and conducted two semistructured interviews with the captain and first officer that were focused around the DP operation and the operator's experience.

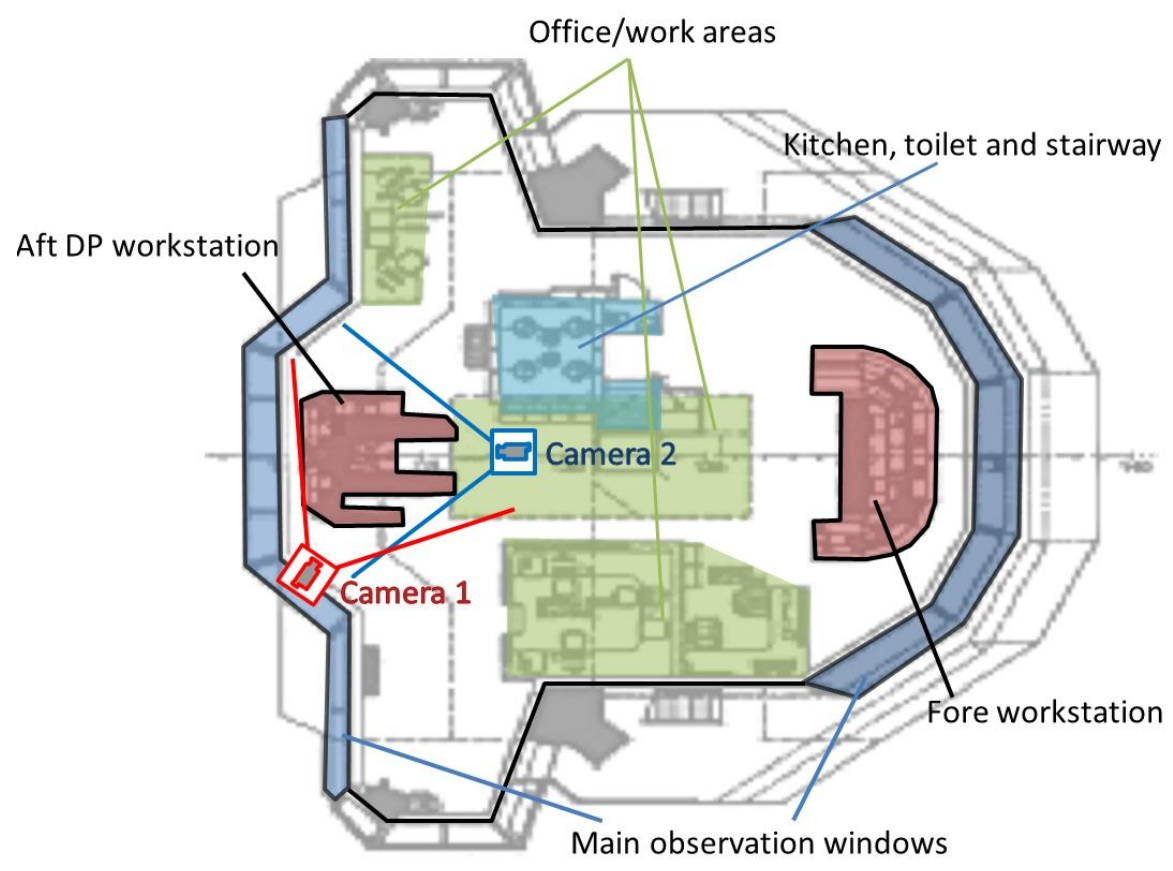

Figure 9: Bridge layout

\subsection{Observations}

Firstly it should be noted that as our observations were conducted on a Norwegian vessel, the leadership structure was almost flat, according to the Scandinavian leadership structure (Buus, 2005), with rank only clearly visible in shift allocation. There have to be two members of the crew on duty on the bridge at all times. Both the captain and the chief officer have one second officer on duty with them and in addition there can sometimes be one midshipman.

Workstations are placed on the aft bridge overlooking the aft deck (see section 2.1.1). Windows run from floor to roof, providing operators with the important view of the aft deck. This view is also where operators' eyes are most of the time apart from when they glance at displays and acknowledge alarms. It was clearly crucial that this view was never blocked. 


\subsubsection{Interview Findings}

The semi-structured interviews provided good insight into the operators' concerns and how they handle everyday struggles like 12 -hour shifts, changing weather conditions, accidents they witnessed and also how they felt about the DP system and accompanying equipment. Issues raised included that they have to stretch out to reach the displays and that the joystick controls become worn out and wobbly. Due to the often low pace and intensity of platform supply operations, operators were sceptical about the benefits of using multi-touch gesture interaction due to that is was novel and felt at this point unfamiliar. However they suggested that it would be beneficial for other types of DP activities, e.g. pipe laying, and remotely operated vehicles.

\subsubsection{Observing the Bridge Environment during Transit}

While the vessel was in transit, we took note of who was on the bridge, communication and movement patterns and possible reminders (post-its or similar) on equipment to ease the operator's mental load. The communication on the bridge was generally between the crew on the bridge and over VHF radio to crew on deck or on shore. When leaving shore, there was extended communication to confirm that everything was ready for pulling out. During steaming, steaming between destinations, and returning to shore, the crew's main tasks were to observe the waters surrounding the vessel and to keep the vessel in normal operation (monitoring equipment status). When reaching the end of the shore zone, the crew radioed the supply base to "check out" from the zone. When returning to shore the same procedure was followed to "check in" and the vessel was allocated a place to berth.

The average time spent steaming was 8 hours, during which activity levels are low. The activity increases between watches, especially for watch handovers, and when the vessel is approaching the goal destination, i.e. the next platform or berthing the vessel at the shore base. When reaching the destination and the 500 meter safety zone around an oil rig, the vessel enters the next phase and prepares for DP operation by going into DP mode.

\subsubsection{DP Operation: Entering the 500m Safety Zone}

We videotaped one operation where the vessel reaches the $500 \mathrm{~m}$ safety zone and goes into DP mode, gets permission from the platform to approach, and then approaches using DP (figure 10). The operator then stabilises the vessel close to the rig, performs supply operations and finishes the operation once the vessel is pulled out from the rig and transfers operation from DP back to normal steaming. DP operations for platform supply purposes last up to several hours depending mostly on the amount of cargo to load/unload.

Within the 500 meter safety zone around an oil rig there are always two operators surveying operation. When handing over command from fore (steaming/piloting) to aft (operations) bridge, the fore bridge gives command to the aft bridge so that the vessel can be fully operated from the aft bridge workstation. When closing in on the rig the vessel is stabilized 
about 10 to 20 meters from the rig. The DP's graphical user interface visualizes the move and often the operator moves the vessel in small stages to ensure it does not get dangerously close to the rig.

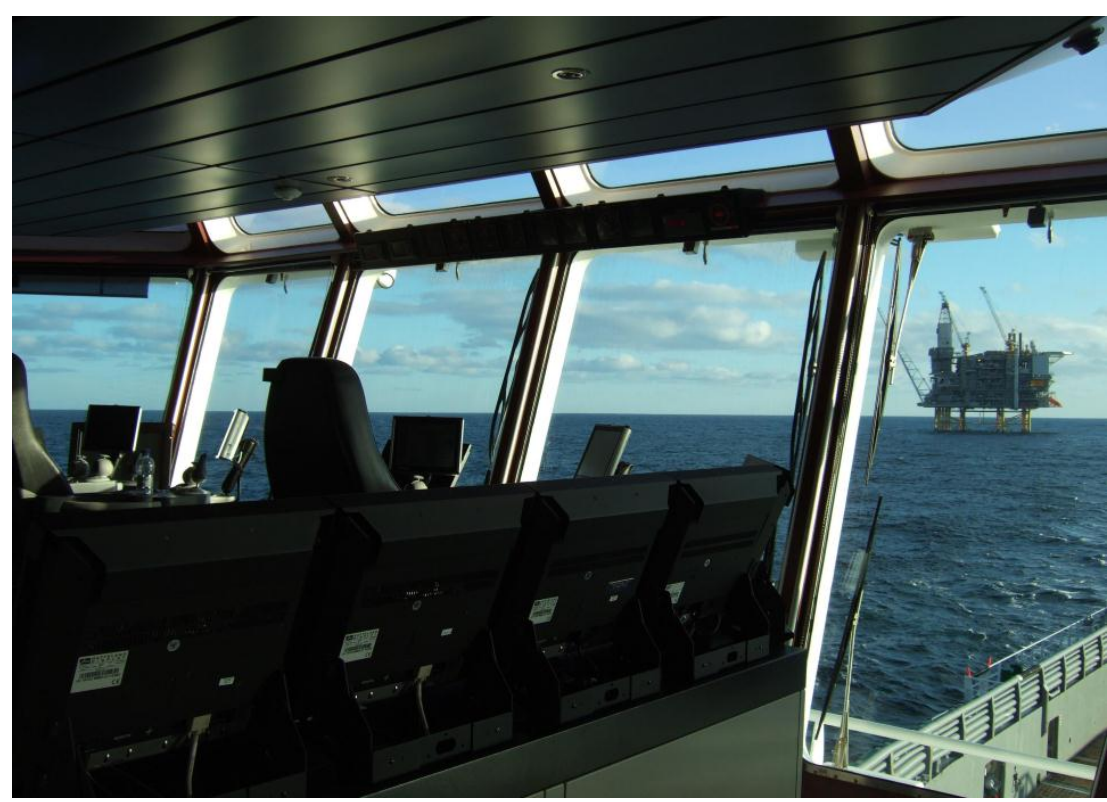

Figure 10: Vessel on 500m safety zone limit

During DP operation, there were few repetitive patterns, apart from following normal procedure, surveying the operation and following requests from the oil rig to come closer or give more distance. Conversations peak in the beginning of an operation and towards the end. During DP operation the deck crew load and offload cargo containers and connect hoses to the tank couplings for pumping liquid cargo.

During operation, the DP operators' responsibility is to survey the operation and the monitoring equipment on the bridge. If there is slack time, they fetch coffee or talk with the crew, but they never stop surveying the aft deck and the monitoring systems. They take turns to counteract fatigue if operations are long and to give less experienced operators practise time. The operator can also be involved in several low cognitive load tasks at the same time with some cross-room communication. If any safety-critical issues, unexpected problems, or breaches of procedure happen (e.g. loss of position/drifting), the operator takes manual control of the vessel by overriding the DP system.

\subsection{Study Discussion and Conclusions}

The observations revealed a well-organised vessel, with an informal and cheerful, but respectful conversation style between crew. This provided a good base of knowledge on how platform supply DP operations at sea are carried out. We discovered that the pace onboard was much lower than anticipated. This can of course vary between different types of DP vessels. The level of stress does, however, increase if weather conditions are bad. 
During DP operation, the DP operator stations and communication equipment were the most frequently used equipment. The system interaction had peak time when closing in on the oilrig. During DP operation, the officer in command of the DP system maintained almost constant view of the aft deck to ensure safety on deck. This confirms the importance of a nonoccluded view raised by expert users (section 3.2.1) and inspired our design for a secondary task in the final experimental study (section 7).

The crew was asked about the possibilities of using multi-touch gesture interaction to operate the system, but were sceptical for platform supply operations since the system today works well. It is worth mentioning that the dynamic approach to the platform, which is often done using the joystick (meter by meter), would be mirrored by multi-touch gesture interaction. Gesture interaction can possibly supplement or replace joystick use. The implications for using multi-touch gesture interaction on a PSV will change the operator station's design, and manoeuvres that can feel strenuous, such as turning their head to look at the displays to the left/right (leaning closer to get a better overview etc.) can be removed.

Our observation study is limited in terms of only one vessel having been observed under normal operating conditions, when most operations follow the same schema. We did not observe operation in bad weather or during significant incidents. For future studies it would be worth doing observations during heavy weather to investigate if the operators behaviour changes and to what extent. However, such observation would have to be very carefully designed as they would involve serious, and legislated, health and safety issues. An observation planned at an earlier date had to be rescheduled when seas went up to 15 meters wave height. Not only would the researcher's safety be at risk in these conditions, but the presence of any additional person on the bridge not belonging to the highly trained crew could be problematic. Furthermore, automatic recording would require a fixed installation of cameras on the bridge, which constitutes an alteration that would have to be confirmed by class regulations.

\section{Two Comparative Experimental Studies}

While raising concerns our observational studies did not rule out multi-touch gesture interaction, as such we moved forward to build on lessons from our paper prototyping and observational studies by developing and testing prototype implementations of the identified user-suggested gestures. Using these prototypes we compared traditional touch button and menu interaction with our multi-touch gesture interactions using a commercial DP system. The aim of these studies was to get a sense of how the gestures extracted from the initial guessability study perform when implemented in a real system. The studies also provided us with user feedback on our designs and iteratively guided the design and implementation of a final system. The final refined prototype was tested on a ship motion simulator located at Aalesund University in Aalesund, Norway. In both experiments we tested 4 of 6 available degrees of freedom (DOF). For the first experiment we tested surge, sway, heave and pitch 
(with yaw and roll being discarded due to technology limitations of the implementation platform). For the second experiment we tested surge, sway, yaw and heave (pitch and roll were discarded as our observations revealed these were not relevant to DP operation in practice).

The remaining empirical work in this paper is based on evaluation of prototypes running on multi-touch screens. Here we introduce the equipment and setup common to both studies. For our research we required a display that detects more than one point of touch, ideally integrated in a slim display with support for all identified gestures, which is close to the style of display currently deployed on DP vessels. For early development and as first prototype a NextWindow optical display was used that supported most tasks, but with limitations on the rotation gesture. The reason behind this was that its optical tracking cannot accurately detect rotation angles due to occlusion of touch points. This can result in the system confusing which $\mathrm{x}$ and $\mathrm{y}$ coordinates belong to the same finger. The second prototype was based on a newly released Dell Windows-7 tablet and supported all gestures.

In both user experiments we compared single-touch interaction against multi-touch interaction interfacing with a commercially available DP system from Rolls-Royce (RR). Both prototypes included code to communicate between the displays, gesture recognition system and the DP control software. The RR DP system's GUI showed an authentic interface with simulated data so that the system responded just like a system on-board a vessel in normal operation, with the single-touch experimental condition being the standard touch interface deployed on DP vessels. The experiments used the RR standard 2D and 3D GUI, where menus are presented in $2 \mathrm{D}$ and the "action" happens in a 3D scene showing the vessel (see figure 2).

In standard operation the DP operator first gives the command for a move and then has to confirm this command as an explicit step (due to the safety-critical nature of the task). After a command is confirmed, it will usually take a while for the motors to initiate action and the vessel to move into the new position. A move is indicated in the GUI using different colours of the vessel. Blue indicates "in position", grey indicates "move initiated, but not confirmed" and yellow indicates "moving to position". The grey and yellow images are shown as a shadow indicating the new position next to the ship.

With the traditional soft-button interface, the operator double taps the desired menu item in the top left of the GUI (figure 2) to initiate the vessel or 3D scene camera movement. Then, a popup window appears with two fields for directional input (one for moving port/starboard and one for moving forward/backward) with a virtual keypad for typing the desired distance (e.g. $15 \mathrm{~m}$ forward and $15 \mathrm{~m}$ starboard). To initiate the move, 'apply' must be pressed. For safety reasons, the apply-button has a 1 second delay before it is activated and turns green. 
Zooming and tilting the 3D scene camera are less safety critical and only require a press and hold with no confirmation.

In the multi-touch gesture interaction condition participants could directly touch the graphical depiction of the vessel and manipulate it in the 3D scene. An additional tap with two fingers was used to confirm or accept the command. For example, the operator drags the vessel (turns grey) using two fingers to the desired position, then two-finger taps to confirm before the vessel starts moving (turns yellow).

\section{First Experiment, first Prototype}

The aim for this study was to test multi-touch gesture interaction in a real Rolls-Royce DP application to investigate performance times.

\subsection{Study Design}

The experiment consisted of four parts: plenary introduction session, individual introduction, series of tasks and a post task discussion. All tasks were videotaped and the timestamp for each operation was recorded. The experiment was conducted in a controlled laboratory environment with eleven participants from the Aalesund University's nautical studies course who had knowledge of DP systems, but no extended experience. The choice of participants was motivated to balance the paper and on-board studies with users who were likely to be less predisposed towards traditional interaction. The subjects were mostly aged between 19 and 21 , with one 25 years old and two 27 year olds.

The key hypothesis investigated here was H1: multi-touch gesture interaction will lead to faster interaction with the DP system. The independent variable was interaction style (button/menu vs gesture) with task completion time being the key dependent variable.

\subsection{Experimental Setup}

Each participant took about 20 minutes with 15 minutes on tasks and 5 minutes for a posttask discussion/walkthrough. Each participant was seated at a table with two displays in front of him/her, one a traditional Rolls-Royce touch display and one a NextWindow-display with multi-touch functionality (see figure 11). Participants were randomly allocated to start with one experimental conditions (counterbalanced). They were given 9 tasks to complete twice in each condition, allowing us to assess learning between first and second attempt. The tasks concerned zooming in/out of the 3D scene, moving the vessel 5 meters forward/backward (surge), 5 meters left/right sideways (sway), tilting the vessel up/down and resetting the vessel to its original size and position. Yaw and roll were discarded from this setup for separate reasons: Yaw was discarded due to constraints with the optical touch technology while roll was discarded since this functionality was not available in the commercial RollsRoyce DP system (indicating it is not relevant for DP operation). 


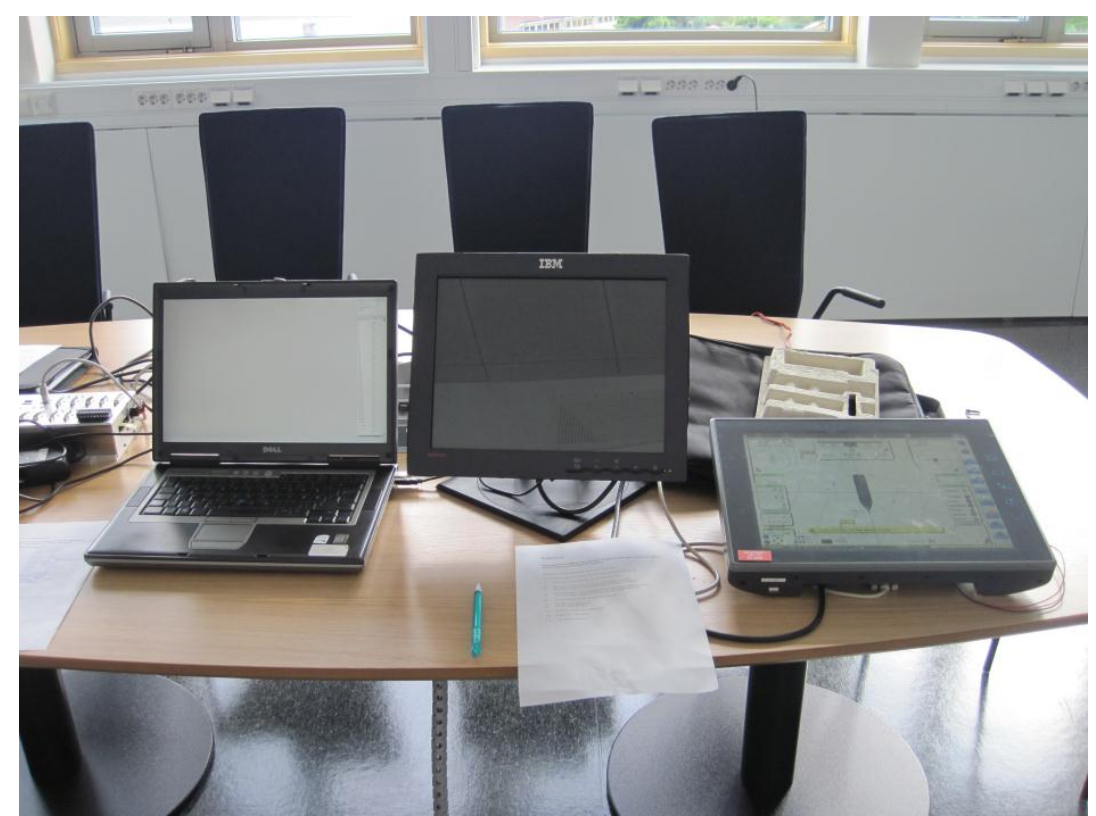

Figure 11: Control laptop, NextWindow multi-touch display, Single touch DP display

Participants had to use two fingers for all tasks (table 3) instead of using one finger for some tasks as suggested in the prototype study (section 3). This was due to technical constraints in the hardware which could not handle swapping between detecting one and two touches but did not seem to have any influence on the user's performance.

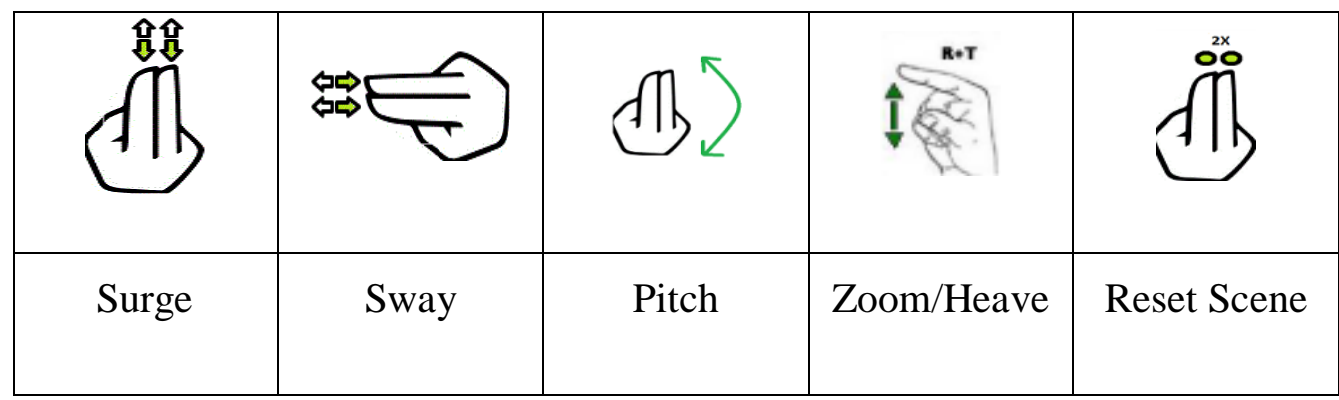

Table 3: Prototype 1 gestures

\subsection{Findings}

Participants did 18 tasks in total $(9 \times 2)$ with multi-touch gesture interaction and touch button and menu interaction. Using the traditional button/menu interface took on average $6.52 \mathrm{~s}$ per task (averaged over all tasks) with a high standard deviation of 6.09s. Using multi-touch gesture interaction it achieved 4.98s mean (SD 3.37s). This difference was significant ( $\mathrm{p}<0.01$, two-tailed paired t-test) and shows that multi-touch gesture interaction performed faster overall. However, as shown in figure 12, this advantage was not uniform. Between the first and second attempt the participants improved with both interactions techniques by around $30 \%$ (statistically significant). 


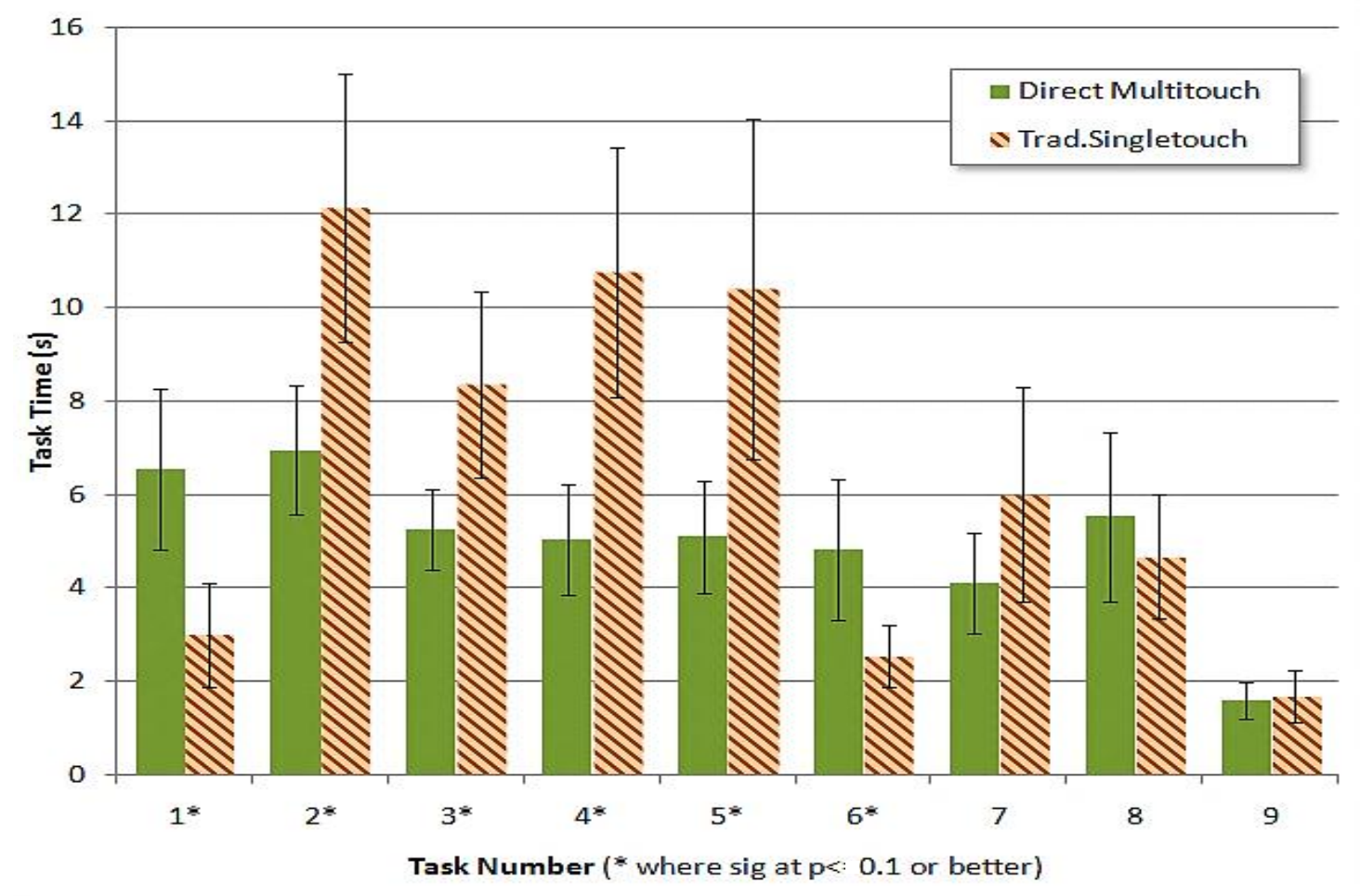

Figure 12: Task times for 1: zoom in, 2/3: surge, 4/5: sway, 6: zoom out, 7/8: pitch, 9: reset

When interacting via gestures, participants seemed to hesitate less. They went straight into moving the vessel, whereas with traditional menu/button based interaction they were searching longer for the right button/menu. During their first attempt using the system (regardless of technique used), they needed some guidance on how to perform the tasks.

One of the participants was left-handed. He found the menu/button-based technique easier than the right-handed participants, due to the menus being on the GUI's left side. The righthanded participants experienced hand occlusion when pressing these buttons and menu selections. This problem was eliminated with multi-touch gesture interaction.

For direct movement (surge and sway), multi-touch gesture interaction proved faster (by $5.16 \mathrm{~s},(\mathrm{p}<0.01)$ for forward and 3.01s $(\mathrm{p}<0.01)$ for aft, 5.74s $(\mathrm{p}<0.01)$ for starboard, $5.23 \mathrm{~s}$, ( $\mathrm{p}<0.05)$ for port). For gesture-based surge, participants had some difficulties to move exactly 5 meters and often deviated by one meter (but no more), and this improved between the first and the second attempt. This indicated that the system might be too sensitive for distance specification. When performing sway operations some participants twisted their hand in an awkward position, indicating that this gesture might be easier to perform with a more horizontal display.

Zooming the scene (heave) was faster with the traditional single touch interaction (by $3.54 \mathrm{~s}$ for zooming out $(\mathrm{p}<0.01$, paired t-test) and 2.28s for zooming in $(\mathrm{p}<0.05)$ ). The participants pressed once or twice on the "zoom in/out"-button, while holding the gesture longer. A general trend was that they mixed up which gesture was zooming in and which was 
zooming out with roughly half of the participants initially zooming in the wrong direction indicating that using the pinch gesture to zoom might not be as natural as widely claimed.

Tilting the scene was very easy for some participants but hard for others, without a consistent difference between the two interaction styles in interaction time. The gesture used was two fingers slightly apart doing a curved vertical movement. This was the gesture least liked by participants, with traditional menu/button based interaction being preferred for this task (but here they occasionally tilted the 3D scene camera the wrong way).

Resetting the scene was a short and easy task without any performance difference between interaction styles.

In the post task discussion participants commented on their impression of the two interaction techniques. The overall opinion was that in general the system was easy to understand but that the GUI for traditional menu/button interaction was cluttered and that the multi-touch technology detected attempted gestures too quickly. About half of the participants preferred button/mouse interaction because it is more accurate, with the other half preferring multitouch gesture interaction since it felt more efficient (provided accuracy issues could be solved). The majority (8) thought that multi-touch gesture interaction is more intuitive and potentially safer. A majority (9) thought that multi-touch technology had the potential to increase efficiency and feeling of control for DP control. Finally, the participants thought that tactile feedback (e.g. Hall, Hoggan and Brewster 2008) could possibly increase the feeling of control when operating the system.

\subsection{Study Discussion and Conclusions}

Multi-touch gesture interaction was faster for simple positioning movements. These are the most frequent commands for DP systems. The tasks found to be slower with multi-touch gesture interaction concerned zooming and tilting upwards. The dynamic nature of the zoom/tilt does however come at a price of time taken, which shows in this user test.

Overall, multi-touch gesture interaction was found to be both faster and, according to comments from the participants during the post-task discussion, more intuitive than traditional button/menu interaction. A majority of participants were optimistic that multitouch gesture interaction is more intuitive, and potentially safer and more efficient than the traditional interface, provided that sensitivity issues could be resolved. Participants felt that the multi-touch display used sensed the gestures made on the display too easily, causing the vessel to move too abruptly. Hypothesis $\mathrm{H} 1$ for this study was supported, with tasks carried out in less time using multi-touch gesture interaction. This is in line with previous research by e.g. Buxton and Myers (1986) and Balakrishnan and Hinckley (1999). However, the set of gestures required fine tuning with decreased sensitivity. 


\section{Study of Refined Prototype on Ship Motion Simulator}

Our final study resulted in our main findings, some of which ran counter to our expectations and earlier results. The final study was run using a motion platform configured to simulate sea conditions on vessels and normally used for training marine personal.

The aim of this study was to test the general performance of multi-touch gesture interaction compared to traditional interaction using a refined prototype based on our iterative design process of gesture study, observational study and first experiment, that addressed the issue of over-sensitivity to gesture input. Given that gesture input for moving the vessel 15 metres requires only a rough selection with subsequent drag plus one double-tap, compared to five to seven menu or button presses (including a double-tap) on the GUI interface with relatively small touch targets, our key hypothesis was that multi-touch gesture interaction would be quicker and easier. The findings made on-board a vessel contributed to a task set that included awareness monitoring and led us to investigate the impact of a moving environment on performance. Prior research using ship motion simulators has indicated that motion can impede fine-motor control (Wertheim, 1997). The motion of a vessel might thus impede users' ability to repeatedly select small targets (menu/buttons), but might also negatively affect the accuracy and timing of multi-touch gesture interaction.

A brief pilot study with eight subjects was carried out to gain experience of evaluation within the motion simulator platform.

\subsection{Main Study Design: Equipment and Motion Environment}

Again, the Rolls-Royce DP system was employed, but now using a Dell tablet computer similar in size to the touch-screen displays used in DP operation. The experiments were carried out in the high-speed craft simulator at Aalesund University. This environment consists of a moving platform designed to simulate sea conditions (heave, pitch and roll) with a realistic bridge setup and a large wrap-around display (figure 13). Emulating the on-board setting, the tablet was raised slightly towards the operator $\left(30^{\circ}\right.$ horizontal). One camera recorded the experiment, monitoring the tablet's surface and immediate context. 


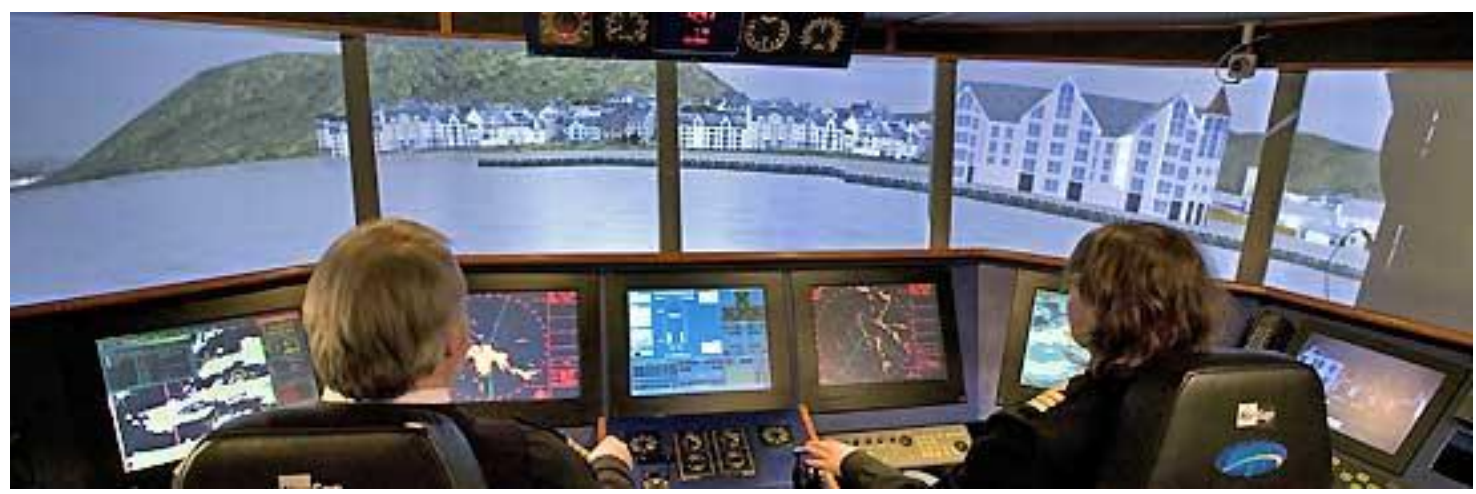

Figure 13: Motion simulator with visualisation of environment

Our at-sea observation had revealed that DP operators need to look out of the windows during operation to ensure safety on deck and around the vessel. Our distractor task emulated this activity and allowed us to measure reaction time to this awareness task. Crossing crafts appeared in the visualisation (on the wraparound display) at specific intervals. Every time a craft was visible, the participant had to shout "boat" or "helicopter". For each session there were six crossings, in addition to seagulls that were to be ignored. The landscape was an authentic visualisation of a well-known strait outside Aalesund Harbour.

\subsection{Experimental Parameters, Participants and Tasks}

Our hypotheses for this study were:

H1: Multi-touch gesture interaction will lead to faster interaction;

H2: Multi-touch gesture interaction will be less erroneous;

H3: Multi-touch gesture interaction will lead to more situational awareness;

H4: Multi-touch gesture interaction will be less affected by a moving environment;

We used a $2 \times 2$ within-subjects study design with interaction style and setting (motion) as independent variables:

IV1: Interaction style: multi-touch gesture and buttons;

IV2: Motion: Static and simulated medium-rough sea.

The participants were first and third year students at Aalesund University studying for degrees within navigation and manoeuvring large vessels. In total 19 students participated (eleven $3^{\text {rd }}$ year and eight $1^{\text {st }}$ year students; seventeen male and two female; aged 20 to 32 , mean age 23; 18 right-handed). Students were recruited for pragmatic reasons, as access to skilled operators is extremely difficult, but also because extended prior experience may predispose users towards the traditional interface, biasing the results. 
First, participants were trained how to use the system. Each session then lasted 20 to 25 minutes. Scripted task instructions were given verbally by the facilitator. Eight different tasks were performed. Tasks were repeated twice in each condition, with the order of conditions counterbalanced. The distractor task scenery (different timings) was also balanced over the experimental conditions. The selection of a distractor task concerning reacting to crossing crafts was based on our observations at sea (see section 4).

The tasks were largely identical with those from the previous study. With the new touch screen hardware, the rotation task (yaw) could now be included. Pitch and roll was discarded as both had been identified as non-crucial for DP operation.

The tasks were:

1. zooming out from the vessel in the $3 \mathrm{D}$ scene,

2. moving it 15 meters forward,

3. moving it 15 meters backward,

4. moving it 15 meters port/left,

5. moving it 15 meters starboard/right,

6. rotating the vessel 7 degrees starboard/right,

7. rotating it 7 degrees port/left,

8. zooming in.

The interactions required for each task were identical with the ones in the first experimental study. The traditional soft-button interface requires double-tapping a menu item, marking a field (indicating direction of the move) and entering the distance on a virtual keypad, followed by pressing 'apply' after one second delay. Zooming consists of press and hold. In the multi-touch gesture interaction condition, the representation of the vessel is manipulated by two-finger gestures, followed by a two-finger double-tap to confirm the action. To move surge and sway the participant put two fingers on the vessel visualised in the GUI and pushes it to the desired position. For rotation one finger is kept stable on the vessel while the other makes a flicking gesture until the counter shows the desired amount of degrees in either port or starboard direction. Zooming is done via the 'pinch' gesture known from the iPhone.

\subsection{Findings}

Time spent per task, error rates and reaction times to incoming vessels were measured. Due to a system crash, data from one session was lost affecting the number of participants in some statistics but without bias. Main data was analysed using repeated-measure ANOVA with significant effects verified using pairwise comparisons with Bonferroni correction, and retested excluding extreme data points (i.e. two fastest and two slowest). 


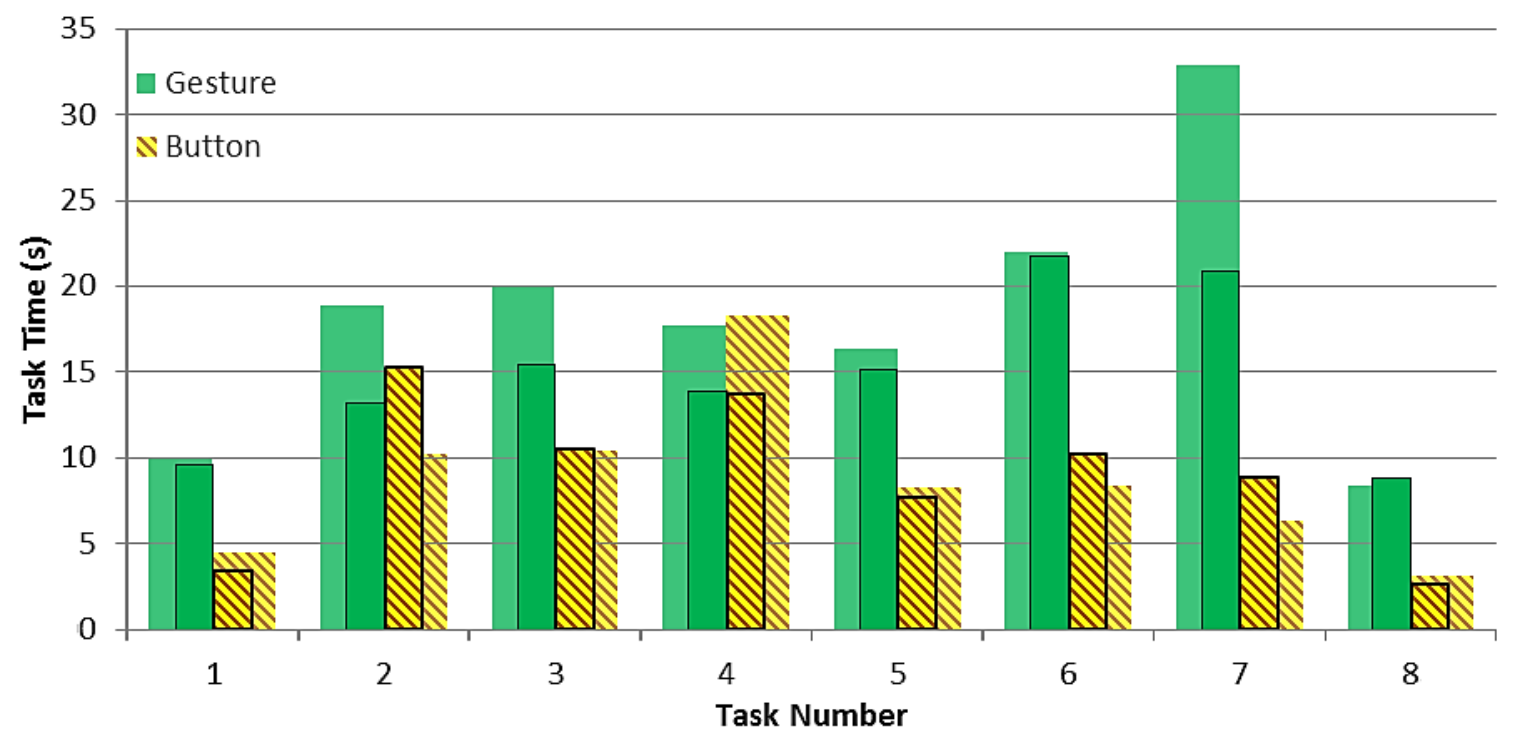

Figure 14: Results on time taken per task (front=static condition, rear=motion)

\subsubsection{Effect of Interaction Style on Time Taken for Tasks}

Figure 14 shows the mean time taken for individual tasks and compares buttons vs gestures in moving and static conditions. Figure 15 shows the mean overall task time (summarized over all tasks) (with 95\% confidence error bars). The difference between gestures and buttons is significant $(\mathrm{F}(1,104)=72.62, \mathrm{p}<0.01)$. Refuting $\mathrm{H} 1$, we found that multi-touch gesture interaction was significantly slower than touch-based button interaction both in general (see figure 15) and for nearly all tasks, some (moving right and both rotation tasks) taking almost twice as long (zoom out: $\mathrm{F}(1,11)=13.36, \mathrm{p}<0.01$; zoom in: $\mathrm{F}(1,12)=14.61, \mathrm{p}<0.01$; move forward: $\mathrm{F}(1,11)=13.365, \mathrm{p}<0.01$; move back: $\mathrm{F}(1,12)=7.502$, $\mathrm{p}<0.05$; move right: $\mathrm{F}(1,6)=29.759, \mathrm{p}<0.01$; rotate right: $\mathrm{F}(1,13)=14.814, \mathrm{p}<0.01$; rotate left: $\mathrm{F}(1,11)=$ 90.014, $\mathrm{p}<0.01$ ). Moving backward ceased being significant when excluding the two slowest and fastest instances from the data, but still showed a trend. Only task 4 (moving left) had no difference. 


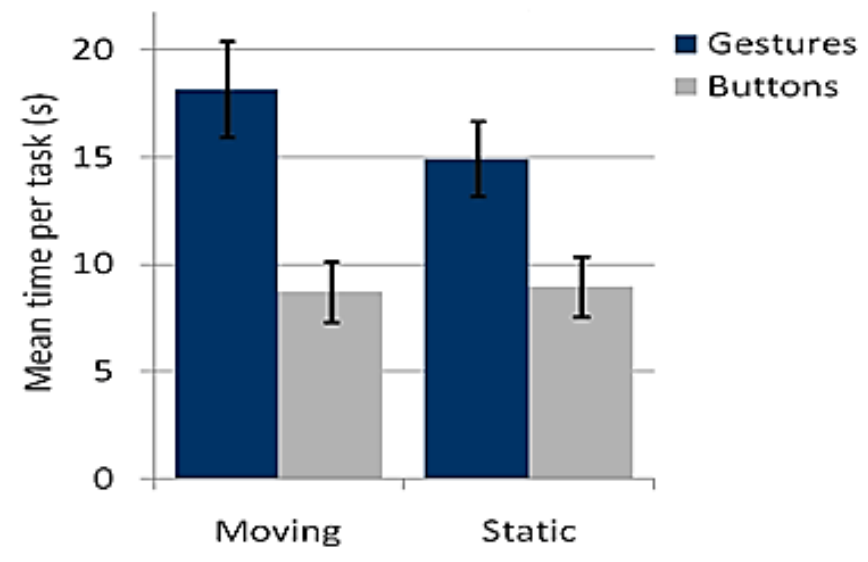

Figure 15: Overall mean task times from motion platform study

We measures reaction time to environmental events as a proxy measure for situational awareness. Paired t-tests showed no significant difference in reaction time to the distractor task between the interaction techniques. Thus hypothesis $\mathrm{H} 3$ is not supported.

\subsubsection{Effect of Interaction Style on Errors}

The effects of interaction style on errors vary with no clear pattern. For moving and rotating, errors in the button condition often seemed to be caused by the long sequence of actions and the delay for 'apply'. An error tended to trigger a series of further errors, indicating increased stress. With gesture input, some participants had problems stopping at the exact distance, moved in the wrong direction at first, or held their fingers too close together for the twintouch gestures to be recognized, and a few failed to complete the command altogether. For rotation and moving sideways, the effects on errors were asymmetrical for left and right.

The gesture for zooming in and out, a.k.a. 'pinch', is well-known from the iPhone and Windows 7 so could be believed to be easy to carry out. However, as already observed in our first experimental study, many users zoomed the wrong way - confusing zoom in and out in $44 \%$ of first gesture attempts. Overall $30 \%$ of zooms were erroneous with gestures but only $3 \%$ tapped the wrong button, a significant difference: $\mathrm{F}(1,11)=4.714, \mathrm{p}<0.05 . \mathrm{H} 2$ is therefore not supported.

\subsubsection{Impact of Motion}

We found a consistent tendency for the simulated vessel movement to slow down interaction. When comparing summary timings for all tasks (figure 15), a main effect of motion on time spent was found $(\mathrm{F}(1,104)=4.25, \mathrm{p}<0.05)$ as well as an interaction between motion and input style on time taken, with motion impeding gesture interaction $(\mathrm{F}(1,104)=7.05$, $\mathrm{p}<$ $0.01)$.

Motion tended to slow down zooming and moving the vessel sideways, and increased the time taken for a left rotation $(\mathrm{F}(1,11)=5.053, \mathrm{p}<0.05)$. When moving forward, multi-touch 
gesture interaction was slightly faster than button interaction in the static situation, and much slower in the moving environment. Both task 2 (move forward) and task 7 (rotate left) had interaction between motion and input (significant only with extreme values) $(\mathrm{F}(1,11)=$ 10.917, $\mathrm{p}<0.01$ and $\mathrm{F}(1,11)=9.885, \mathrm{p}<0.01)$, with multi-touch gesture interaction taking on average 32 seconds during motion, but only 20 when static. We found little impact of motion on error, only leftward moves and rotation were affected. H4 is therefore not supported.

\subsubsection{Potential Handedness Effects - Asymmetries in Findings}

Our findings are asymmetric for some tasks, for our predominantly right-handed users. Motion affected moving forward via gestures more than moving backwards. Moving the vessel left had no difference between gesture and button input. But for moving right, touch button interaction performed almost twice as fast as gesture $(F(1,6)=29.759, p<0.01)$. For rotating and moving sideways the effects on error were also asymmetric. When rotating left, participants made significantly more errors using gestures $(F(1,11)=6.769$, p < 0.05) but tended to make less errors with gestures when rotating right. For moving left, buttons tended to be much more erroneous $(\mathrm{F}(1.12)=4.347, \mathrm{p}<0.10)$, while for moving right gestures tended to result in more errors $(\mathrm{F}(1.15)=4.233$, $\mathrm{p}<0.10)$.

Motion in particular seemed to only effect gestural rotation to one side. For leftward rotation an interaction between motion and input on time taken was found when including extreme values $(\mathrm{F}(1,11)=9.885, \mathrm{p}<0.01)$. Furthermore there was significant effect of motion on error $(\mathrm{F}(1,11)=6.769, \mathrm{p}<0.05)$ and a significant interaction between motion and input on error $(\mathrm{F}(1,11)=5.077, \mathrm{p}<0.05)$. All of this effected gestural input to become slower and more erroneous with motion (gesture errors tripled from 0.4 to 1.2 with motion).

The 'move right' gesture and pivotal left rotation, thus seem harder to execute than the other direction. Especially the sideways flick seems difficult, and a few participants tried to use the other hand. This is a strong indication that handedness can be an issue for the usability of touch gestures.

\subsubsection{Other Findings}

The videos revealed that the interaction style influenced how participants dealt with the distractor task. When interacting via gesture, the majority of participants kept their fingers on the surface, continuing a gesture whilst looking out of the window. With buttons, participants paused to look out, and then looked down again to reorient. This indicates that gesture interaction may be advantageous for situation awareness and for applications that require continuous input.

On a post-study questionnaire with 7-point Likert-scaled questions participants clearly preferred button-based interaction over gestures, with opinions strongest for rotate $(2.21$ 
rating). Only a small subgroup found gesture input easier. Gesture input scored highest for zooming (3.61). Interestingly, participants seemed to forget about the errors that had occurred with touch buttons/menus under stress more easily compared to the difficulty experienced when moving an accurate distance via gestures.

\subsection{Summary of Findings}

Counter to expectations, we found that the soft-button interface outperformed multi-touch gesture interaction (figure 15). Some of these performance issues might be due to our tweaking of the surface sensitivity to touch. But this was done in response to user complaints about the prior prototype being too sensitive. In addition, we found that environmental motion effects gesture input negatively. This was despite the fact that the kind of motion generated by the motion platform simulates the motion of a large vessel. This movement is a rather gentle and rhythmic (thus predictable) roll, rather than the kinds of rocking experienced on a small boat.

We also found evidence that the pinch gesture is problematic and its direction frequently confused. Moreover, we found asymmetric results for directional gestures, with gestures in one direction being easier and quicker to perform then in the other direction. Observations indicated a potential positive effect of gesture interaction, enabling participants to suspend and continue gesture input fluidly to look up, with less time needed to reorient.

\section{Discussion and Conclusions}

The aim of this research was to test four main hypotheses concerning the enhancement of accuracy and safety during dynamic positioning operations. These were compared to traditional menu/button DP operation:

- H1: Multi-touch gesture interaction will lead to faster interaction.

- H2: Multi-touch gesture interaction will be less erroneous.

- H3: Multi-touch gesture interaction will lead to more situational awareness.

- H4: Multi-touch gesture interaction will be less affected by a moving environment.

Most multi-touch research compares variations of direct manipulation or mouse with direct touch input (e.g. Forelines et al, 2007). Developing an alternative for an existing commercial touch-screen system had us compare menu/button GUI touch interaction with direct manipulation multi-touch gesture interaction. The literature indicates that touch interaction is not suited for precision tasks and that menu interaction is not suitable for touch interaction (Lepinski et al., 2010). In the DP system used here, menu/button interaction requires multiple selections of relatively small targets and may suffer from occlusion problems (cf. Forelines et 
al. (2007), Kin et al.(2009)) and multiple target acquisition delays. Our hypothesis was thus that multi-touch gesture interaction would perform better than the soft-button interface.

Four studies were conducted, one of these an observational study onboard a platform supply vessel in the North Sea. We initially used paper prototypes to investigate which gestures are most commonly suggested for typical DP tasks. The design of our gesture set followed a similar approach as (Wobbrock et al., 2009). Given this is a specialist interface, we had expert users (DP operators and designers) suggest gestures they would find intuitive. To initiate our investigation of the four hypotheses we identified the most common gestures that could be implemented without ambiguities and eliminated any gestures that would be difficult to execute consistently. Most of these gestures are fairly similar to those used in common multi-touch environments (pinch, drag etc.) (GestureWorks, 2010).

The remaining work involved two hardware iterations and three revisions of the control software. The results from each prototype study plus observations from on-board PSV Havila Foresight provided the base for the next iteration. To investigate H1 we first compared multitouch gesture interaction versus touch button and menu interaction in a static laboratory environment on a prototype using optical touch technology. The final prototype, running on a tablet computer, was tested in a semi-realistic simulated environment, using a ship motion simulator. Issues discovered in the previous study were addressed in the final prototype implementation and experiment design. In the final evaluation to investigate $\mathrm{H} 1$ and $\mathrm{H} 4$, performance of touch menu-and-button interaction as well as of multi-touch gesture interaction was tested both in a moving and a static environment. Interaction error rates and reaction time to cognitive distractions tasks were recorded, to look into $\mathrm{H} 2$ and $\mathrm{H} 3$.

While gesture input had been faster in the first experimental comparison study, it was significantly slower in the second study. This is likely due to our changes to gesture recognition. But these were in response to user complaints about the first prototype reacting too sensitive to gesture input, resulting in abrupt movements of the vessel icon, which made it difficult to accurately move the vessel for specified amounts. This indicates a trade-off between speed of interaction (sensitivity to touch), input accuracy and users' feeling of being in control.

Despite following a careful user-centred design process with several prototypes and a comparison with a traditional interface that requires rather lengthy interaction and menu selection sequences, our final study showed that traditional interaction was quicker and less erroneous, hence $\mathrm{H} 1$ and $\mathrm{H} 2$ cannot be supported. Furthermore, and against our expectations, simulated sea motion in the moving environment accentuated this difference (compared to a static environment baseline) with multi-touch gesture interaction being affected more negatively than traditional interaction. This shows that hypothesis $\mathrm{H} 4$ cannot be supported either. We also observed initial problems with gestures where users would gesture the wrong 
way, and handedness asymmetries on some gestures that were faster in one direction than the symmetrical opposite. Our key results on motion and handedness are both novel and important warnings for the design of multi-touch gestures in many domains. However, on the positive side, our results show that users were able to suspend gestural interaction more naturally during interruptions than they could with button interaction, thus improving their ability to temporarily suspend tasks for situational observation (a key aspect highlighted in our observational studies). This shows that H3 cannot be supported, since there were no significant differences in reaction time for distractor tasks. However the discovery of a more natural suspension of interaction when using multi-touch gesture interaction can give more situational awareness.

Regarding the original application area our contribution lies in conducting a user study under semi-realistic conditions that leaves a rather sceptical picture of the utility of multi-touch gesture interaction for this particular task. More generally, we have provided an example where a traditional button interface, despite several shortcomings, unexpectedly performs better than the gesture alternative, despite its careful iterative design. We have shown that environmental motion effects gesture input negatively, a result that could affect many areas including the use of gestures to control and enter text on mobile phones - we are planning more studies on this (building on e.g., Brewster et al., 2007). This effect was noticeable despite the rather gentle and predictable nature of the simulated ship movement. Our asymmetric results for directional gestures indicate that more research is needed on the ergonomics of gestures, and our findings demonstrate that even the well-known pinch gesture is anything but intuitive, exemplifying Don Norman's statement (2010) that gestural interaction is not natural.

\section{Future Work}

Building on the findings from this project, further studies of vessels and equipment interaction will be carried out with the main motivation of finding interaction techniques that can benefit the user experience onboard. In addition it would be interesting to investigate in more detail how the operators interact with the system, equipment, each other and the crew on the aft deck. This research will be carried out through extensive studies using advanced eye-tracking equipment in both a simulated and real environment.

\section{Acknowledgements}

We would like to thank the many people who helped our studies - in particular the participants in all user studies, the crew of PSV Havila Foresight and the staff at the Aalesund University Motion Platform. Figure 8 is provided with courtesy to Havila Shipping ASA. Figure 13 is provided courtesy of Aalesund University. 


\section{References}

Balakrishnan, R. and Hinckley, K. (1999). The Role of Kinesthetic Reference Frames inTwo-Handed Input Performance. Proc. of UIST'99. ACM. 171 - 178.

Bjørneseth F.B., Dunlop, M.D., Hornecker, E. (2010). Assessing the Effectiveness of Multi-Touch Interfaces for DP Operation. Proceedings of International Conference Human Performance at Sea. Glasgow, Scotland. 243-255.

Bjørneseth F.B., Dunlop, M.D., Strand, J.P. (2008). Dynamic Positioning SystemsUsability and Interaction Styles. Proceedings of NordiCHI'08. 43-52.

Bray, D. (2003), Dynamic Positioning, 2nd Edition. Oilfield Publications Inc.

Brewster, S., Chohan, F. Brown, L. (2007) Tactile Feedback for Mobile Interactions. Proc. of CHI'07. ACM. 159-162

Bryman, A., (2008), Social Research Methods ( $3^{\text {rd }}$ edition), Oxford University Press

Buus, I. (2005). Leadership Development: A Scandinavian Model. M Knowledge. Retrieved from Mannaz Global Leadership Development website. Last accessed: 06.07.11 http://www.mannaz.com/articles/leadership-development-a-scandinavian-model

Buxton, W. and Myers, B. (1986). A Study in Two-Handed Input. Proc. of CHI1986. ACM. 321- 326.

Buxton. B. (2007), Multi-Touch Systems that I Have Known and Loved. http://www.billbuxton.com/multitouchOverview.html original Jan 12 2007, updated March 21, 2011

Fitzmaurice, G., Buxton (1997), W. An Empirical Evaluation of Graspable User Interfaces. Proc. of CHI'97. ACM Press, 1997. 43-50.

Forlines, C., Wigdor, D., Shen, C. and Balakrishnan, R. (2007). Direct-Touch vs. Mouse Input for Tabletop Displays. Proc. of CHI'07. ACM. 647-656.

Galliers, J., Sutcliffe, A. and Minocha, S. (1999). An impact analysis method for safetycritical user interface design. ACM Transactions on Human Computer Interaction (TOCHI), Vol 6(4). $341-369$.

GestureWorks (2011).

Last accessed: 05.06.11.http://www.flickr.com/photos/ideum/5241510881/

Han, J. Y. (2005). Low-Cost Multi-Touch Sensing through Frustrated Total Internal Reflection. Proceedings of the 18th Annual ACM Symposium on User Interface Software and Technology UIST'05. ACM Press. 115-118.

Hancock, M., Carpendale, S., and Cockburn, A, (2007). Shallow- Depth 3D Interaction: Design and Evaluation of One-, Two- and Three-Touch Techniques. Proc. of CHI 2007. ACM, 1147 - 1156. 
Hoggan, E., Brewster, S.A., and Johnston, J. (2008). Investigating the effectiveness of tactile feedback for mobile touchscreens. Proc. of CHI '08, ACM, 1573-1582.

Jordan, B. and Henderson, A., (1995), Interaction Analysis: Foundations and Practice, Journal of the Learning Sciences, Vol. 4 (1), 39-103.

Kin, K., Agrawala, M., DeRose, T. (2009). Determining the Benefits of Direct-Touch, Bimanual, and Multifinger Input on a Multitouch Workstation, Proc. of Graphics Interface 2009, Canadian Information Processing Society. 119-124.

Kirk, D., Sellen, A., Taylor, S., Villar, N. and Izadi, S. (2009) Putting the Physical into the Digital: Issues in Designing Hybrid Interactive Surfaces. Proceeding of British HCI 2009. ACM DL. 35-44

Lazet, A., Schuffel, H. (1977) Some Applications of Human Engineering to Wheelhouse Design. pp. 77-86. Vol. 30(7).

Lee, S.K. Buxton, W., and Smith, K.C. (1985). A Multi-Touch Three Dimensional TouchSensitive Tablet. Proc. of CHI'85. ACM. $21-25$.

Lepinski, G.J., Grossman, T., and Fitzmaurice, G. (2010). The Design and Evaluation of Multitouch Marking Menus. Proc. of CHI'10, ACM. 2233-2242.

LLoyd's Register. (2007).World Casuality Statistics. s.1. : LLoyd's Register Fairplay.

MacKay, W.E. (1999). Is Paper Safer? The Role of Paper Flight Strips in Air Traffic Control, ACM Transactions on Human Computer Interaction TOCHI, Vol. 6(4). 311 340 .

Mehta, N. (1982). A Flexible Machine Interface, M.A.Sc. Thesis, Dept. of Electrical Engineering, University of Toronto supervised by Professor K.C. Smith

Micire, M., Desai, M., Courtemanche, A., Tsui, K.M. and Yanco, H.A. (2009), Analysis of Natural Gestures for Controlling Robot Teams on Multi-Touch Tabletop Surfaces. Proc.of Interactive Tabletops and Surfaces ITS'09, ACM, 41-48.

Mills, S. (2005). Designing Usable Marine Interfaces: Some Issues and Constraints. The Journal of Navigation. 58. $67-75$.

Morris, M.R., Wobbrock, J.O. and Wilson, A.D. (2010). Understanding users' preferences for surface gestures. Proceedings of Graphics Interface (GI '10). Canadian Information Processing Society, pp. 261-268

Nielsen, M., Störring, M., Moeslund, T.B. and Granum, E. (2003), A Procedure for Developing Intuitive and Ergonomic Gestures Interfaces for HCI. Int'l Gesture Workshop, 409-420.

Nielsen, D. and Jungnickel (2003), Maritime Accident Investigation and Temporal Determinants of Maritime Accidents: A Case Study. WMU Journal of Maritime Affairs, Vol. 2, No.1, 49-59 
Nishino, H. Utsumiya, K., Kuraoka, D., Yoshioka, K. and Korida, K. (1997). Interactive Two- Handed Gesture Interface in 3D Virtual Environments. Proc. of ACM Symposium on Virtual reality software and technology (VRST '97), ACM. 1 - 8.

Norman, D.A. (2010). Natural User Interfaces Are Not Natural. Interactions. Vol. 17 (3). 610. ACM

Prison J., Lützhöft M., and Porathe T. (2009) Ship Sense- What Is It and How Does One Get It? London : The Royal Institution of Naval Architects, 127- 130.

Reason, J. (1990) Human Error. Cambridge : Cambridge University Press.

Redmill, F. and Rajan, J. (1997). Human Factors in Safety Critical Systems. Oxford: Butterworth Heinemann.

Shea I.P., and Grady N., (1998). Shipboard Organizational Culture in the Merchant Marine Industry, Proceedings of the Safe Navigation Beyond, Gdynia.

Sillitoe A.,Walker O. and Earthy J. (2009) The Case for Addressing The Human Element in Design and Build. London : Royal Institution of Naval Architects. 19-27.

Smith, M.J., Salvendy, G., Harris, D. and Koubek, R.J. (2001). Usability Evaluation and Interface Design: Cognitive Engineering, Intelligent Agents and Virtual Reality, Vol. 1, pp. 1421. Lawrence Erlbaum Assosciates, Inc., Publishers.

Song, Y. (2009), Analyzing Human Error Triggered Accidents On board Ships Against Ergonomic Design Principles. London : The Royal Institution of Naval Architects. 97-104.

Squire, D.(2009) Keeping the Human Element in Mind. London : Royal Institution of Naval Architects. 1-7.

Wertheim, A. H. (1998). Working in a Moving Environment. Ergonomics 41(12). 18451858 .

Wertheim, A. H. and Kistemaker, J. A. (1997), Task performance during simulated ship movements. TNO-TM report TM-97-A014, TNO Human Factors Research Institute, Soesterberg. The Netherlands.

Wobbrock, J.O., Morris, M.R. and Wilson, A.D. (2009). User-Defined Gestures for Surface Computing. Proc. of CHI'09. ACM, 1083-1092. 